\title{
The dissociative psychedelic renaissance
}

\author{
Sanjay J. Mathew and Carlos A. Zarate (Eds.) \\ Ketamine for treatment-resistant depression: The first decade of progress \\ Adis, Springer Nature, Cham, Switzerland, 2016, 155 p. \\ Hardback ISBN: 978-3-319-42923-6
}

Phil Wolfson and Glenn Hartelius (Eds.)

The ketamine papers: Science, therapy and transformation

Multidisciplinary Association for Psychedelic Studies, Santa Cruz, CA, 2016, 429 p.

Paperback ISBN: 0-9982765-0-2

With all the recent excitement about the resurgence of clinical research on the classic serotonergic psychedelics, in what has been labeled the psychedelic renaissance, it is easy to overlook a concurrent revolution taking place in the field of ketamine research. More than 50 years have passed since the synthesis of ketamine, and it remains today the most widely available compound with psychedelic properties - nearly ubiquitously approved for both research and clinical use. Ketamine has recently proved to be highly effective for depression and suicidality, with a rapid onset of effect. Despite currently being the leading cause of disability in the world, there have been no major advances in depression treatment, since the selective serotonin reuptake inhibitors of the 1980s. In the context of this stagnation, research on ketamine has been met with understandable enthusiasm, typified by former director of the National Institute of Mental Health (NIMH) Thomas Insel's proclamation that ketamine "might be the most important breakthrough in antidepressant treatment in decades."

Two books, Ketamine for Treatment-Resistant Depression: The First Decade of Progress edited by Sanjay J. Mathew and Carlos A. Zarate, Adis, 2016, and The Ketamine Papers: Science, Therapy and Transformation edited by Phil Wolfson and Glenn Hartelius, Multidisciplinary Association for Psychedelic Studies, 2016, explore in depth the fascinating history and clinical background of using ketamine for depression. These two books are the most comprehensive works to date that summarize the progress made on ketamine research in the field of mental health. While both cover the major milestones in the history of ketamine's development, safety, and clinical data, they have substantially different approaches and foci. They ultimately complement one another, each filling in gaps where the other is more cursory. Both Ketamine for TRD and The Ketamine Papers are essential reading for any researcher or health care provider interested in understanding the history and science behind the use of ketamine for the treatment of mental health disorders.

Ketamine is an arylcyclohexylamine derivative that is primarily a non-competitive N-methyl-D-aspartate (NMDA) glutamate receptor antagonist. It was first synthesized in 1962 to replace phencyclidine as a surgical anesthetic with a better safety profile and fewer adverse effects. It was tested on human prisoners in 1964, FDA approved in 1970, and subsequently gained widespread use for battlefield anesthesia during the Vietnam War.

Ketamine's unique psychoactive effects at subanesthetic doses were noted early on by Edward Domino during his first experiments on humans. Patients reported both significant changes in mood as well as a sense of "disconnection" from their bodies, which led him to coin a new and unique drug class: "dissociative." Ketamine is now classified as the penultimate dissociative anesthetic, describing the dosedependent continuum leading to eventual dissociation of the conscious state from awareness of the physical self prior to complete anesthesia. At a subanesthetic dose, ketamine reliably produces alterations in consciousness, including positive mood, altered perception and cognition, transcendence of space and time, a sense of awe, ineffability, depersonalization, and derealization often coexistent with a conscious awareness of a sense of unity. These psychoactive effects were not lost on psychedelic researchers, as the FDA approval of ketamine in 1970 coincided with the global shutdown of research on the classic serotonergic agents as a consequence of the 1971 UN Convention on Psychotropic Substances.

Beginning in the 1970s, Khorramzadeh and Lofty (1973) and Roquet, Favreau, Ocana, and Velasco (1975) began to experiment with ketamine in the tradition of drug-assisted psychotherapy as narco-analysis, abreactive, psycholytic, and psychedelic therapy, describing cases of sustained psychological benefits and improved mood using psychoactive doses. While The Ketamine Papers dedicates a full six chapters to this first stage of research and clinical exploration, Ketamine for TRD mentions that same early work over several decades in just one paragraph of the book (even announcing this omission in its subtitle "The First Decade of Progress" a full 20 years after initial reports of clinical use). The Ketamine Papers starts the story with formal clinical trials begun in the 1990's. To some extent, the different approaches could be tracked to the semantic history of early psychedelic research. Whereas The Ketamine Papers both acknowledges and explores the potential "mind-manifesting" properties of ketamine (in the

This is an open-access article distributed under the terms of the Creative Commons Attribution-NonCommercial 4.0 International License, which permits unrestricted use, distribution, and reproduction in any medium for non-commercial purposes, provided the original author and source are credited, a link to the CC License is provided, and changes - if any - are indicated. 
spirit of Humphry Osmond's letter to Aldous Huxley coining the term "psychedelic") as potentially useful therapeutic tools, Ketamine for TRD mostly ignores psychedelic effects or refers to psychoactive changes other than improved mood as psychotomimetic side effects, relegating them to discussions of safety or intolerance of the therapy. This reflects a broader discomfort in mainstream academic psychiatry with the concept of treating mental health conditions with single doses of powerful consciousness-altering substances rather than chronic daily therapy with mood-altering agents.

The Ketamine Papers presents decades of clinical experience with the use of ketamine, but rather than focusing solely on academic research, it emphasizes the transformative potential and phenomenology of the ketamine experience. A co-founder of the Heffter Research Institute and subsequent collaborator with the Multidisciplinary Association for Psychedelic Studies, Phil Wolfson was part of the continuation of psychedelic research and clinical work with ketamine. He has helped to bridge the gap between the pre-prohibition days and the current renaissance in psychedelic-assisted treatment of psychiatric disorders. Contributors who provide first-hand accounts of this early history include the pioneers Stanislav Grof, Ralph Metzner, Eli Kolp, and Evgeny Krupitsky. The Ketamine Papers spends an additional four chapters exploring the transpersonal and transformative potential of ketamine, contrasting with a more neurobiological approach taken by Ketamine for TRD.

Ketamine for TRD starts the journey several decades later when the studies using more modern evidence-based criteria for the conduct of clinical trials began to appear after Krystal et al. (1994) at Yale described the psychoactive effects in healthy volunteers in 1994. This was not intended to characterize any putative beneficial effects of ketamine, but rather the psychotomimetic effects in an effort to elucidate the neurobiology of schizophrenia. Much of the later groundbreaking work at Yale and the NIMH would often follow that lead by characterizing ketamine-induced changes in consciousness as unwanted side effects rather than potentially useful areas of study or tools for psychotherapeutic discovery.

The first small randomized controlled trial using ketamine for the treatment of depression was conducted by Berman et al. (2000) at Yale. It was subsequently replicated by Zarate et al. (2006) at the NIMH in 2006, reporting a very large effect size $(d=1.46)$ with a response (defined as $>50 \%$ reduction in depression scores) of $71 \%$ and remission rate of $29 \%$ within $24 \mathrm{hr}$. Similar results, staggering in comparison to standard antidepressant treatment, have since been replicated in thousands of patients. What Ketamine for $T R D$ might lack in its neglect of the psychedelic properties of ketamine, it makes up for in its thorough and in depth analysis of the preclinical and clinical studies on ketamine in the treatment of depression. Many of the chapters are written by authors of seminal works in the field, including Zarate, James Murrough who demonstrated the extended durability of effect using multiple treatments, Rebecca Price exploring rapid antisuicidal effects, Gerard Sanacora who led the American Psychiatric Association's consensus statement regarding ketamine and many others.

The antidepressant mechanism of action of ketamine is complex and continues to be an active area of research. At subdissociative doses, ketamine blocks the NMDA receptor on subcortical inhibitory $\gamma$-aminobutyric acid interneurons, thereby increasing glutamate release from cortical pyramidal cells. This glutamate release leads to cortical NMDA receptor stimulation but preferentially stimulates the $\alpha$-amino-3-hydroxy-5-methyl-4-isoxazolepropionic acid receptor, which promotes the formation of brainderived neurotrophic factor (BDNF). Increased BDNF is considered to be a source of observed synaptogenesis and increased neuroplasticity. Other mechanisms include rapid normalization of dysfunctioning brain networks, including the default-mode network, and inhibition of the negative reward network in the lateral habenula. Ketamine for TRD goes quite a bit deeper into the neurobiology of ketamine and research exploring these putative mechanisms of action, with the exception that The Ketamine Papers gives more attention to the discussion of whether the higher-level transcendental experience in itself might induce durable therapeutic changes, analogous to the "inverse PTSD" described in research with psilocybin (Garcia-Romeu, Griffiths, \& Johnson, 2014).

In the setting of such a high global prevalence of depression, both Ketamine for TRD and The Ketamine Papers are a must-read for those interested in a thorough and comprehensive review of the history and current status of the use of ketamine to improve our options for the treatment of depression and other mental health conditions, which are sorely lacking. The works complement each other well, and are highly recommended reads for researchers, clinicians, and others seeking to gain insight into the fascinating field of this parallel "dissociative" psychedelic renaissance.

\section{REFERENCES}

Berman, R. M., Cappiello, A., Anand, A., Oren, D. A., Heninger, G. R., Charney, D. S., \& Krystal, J. H. (2000). Antidepressant effects of ketamine in depressed patients. Biological Psychiatry, 47(4), 351-354.

Garcia-Romeu, A., Griffiths, R. R., \& Johnson, M. W. (2014). Psilocybin-occasioned mystical experiences in the treatment of tobacco addiction. Current Drug Abuse Reviews, 7(3), 157-164. doi:10.2174/1874473708666150107121331

Khorramzadeh, E., \& Lotfy, A. O. (1973). The use of ketamine in psychiatry. Psychosomatics, 14(6), 14-346. doi:10.1016/ S0033-3182(73)71306-2

Krystal, J. H., Karper, L. P., Seibyl, J. P., Freeman, G. K., Delaney, R., Bremner, J. D., Heninger, G. R., Bowers, M. B., \& Charney, D. S. (1994). Subanesthetic effects of the noncompetitive NMDA antagonist, ketamine, in humans: Psychotomimetic, perceptual, cognitive, and neuroendocrine responses. Archives of general psychiatry, 51(3), 199-214. 
Roquet, S., Favreau, P., Ocana, R., \& Velasco, M. (1975). The existential through psychodisleptics $-A$ new psychotherapy. Paper presented at "Humanistic Psychology in the Americas" at the Sixth International Conference of the Association for Humanistic Psycho, Instituto de Psicosintesis of the Albert Schweitzer Association, Cuernavaca, Mexico.

Zarate, C. A., Singh, J. B., Carlson, P. J., Brutsche, N. E., Ameli, R., Luckenbaugh, D. A., Charney, D. S., \& Manji, H. K. (2006). A randomized trial of an N-methyl-D-aspartate antagonist in treatment-resistant major depression. Archives of General Psychiatry, 63(8), 856-864. doi:10.1001/archpsyc.63.8.856

Lowan H. Stewart, MD

Medical Director

The Santa Fe Ketamine Clinic, Santa Fe, NM, USA

The Axon Clinic, Oslo, Norway

E-mail: Lowan42@gmail.com 